\title{
Use of fertility treatments in BRCA1/2 mutation carriers and risk for ovarian and breast cancer: a systematic review
}

\author{
D. Huber ${ }^{1} \cdot$ S. Seitz ${ }^{1} \cdot$ K. Kast ${ }^{2} \cdot$ G. Emons ${ }^{3} \cdot$ O. Ortmann ${ }^{1}$ (I)
}

Received: 30 March 2020 / Accepted: 8 July 2020 / Published online: 27 July 2020

(c) The Author(s) 2020

\begin{abstract}
Purpose Mutations in the genes $B R C A 1$ and $B R C A 2$ represent a significant risk factor for ovarian and breast cancer. With increasing number and success rates, fertility protection and treatment are gaining importance also for $B R C A 1 / 2$ mutation carriers. However, the effect on primary cancer risk and risk for recurrence remains unclear. This review analyses the published data on fertility treatment and risk of ovarian and breast cancer in BRCA1/2 mutation carriers.

Methods In this review, we included all relevant articles published in English from 1995 to 2018. Literature was identified through a search on PubMed and Cochrane Library.

Results We identified one retrospective cohort and one case-control study regarding the association of fertility treatments and ovarian cancer risk in BRCA mutation carriers. The studies show no increase in ovarian cancer risk. Furthermore, one case-control study on the association between fertility treatment and breast cancer risk in BRCA mutation carriers and one prospective cohort study on the long-term safety of medication used for fertility preservation in women with a history of breast cancer were identified. One of the studies shows a possible adverse effect for gonadotropin-containing medication.

Conclusion Possible increases in cancer risk associated with fertility treatments in BRCA1/2 mutation carriers cannot be excluded at this time. Based on the existing studies, BRCA1/2 mutation carriers should not be generally excluded from fertility treatments. However, they have to be informed about limited data and possible increases in cancer risk.
\end{abstract}

Keywords Fertility treatment $\cdot$ Fertility preservation $\cdot$ Breast cancer $\cdot$ Ovarian cancer $\cdot B R C A 1 \cdot B R C A 2$

\section{Introduction}

In the last decades, the number of fertility treatments is increasing due to postponing family planning to a later age. Improved techniques have led to enhanced success rates and social acceptance is high. A possible association between fertility treatments and ovarian cancer has been described.

D. Huber and S. Seitz contributed equally.

O. Ortmann

olaf.ortmann@klinik.uni-regensburg.de

1 Department of Gynecology and Obstetrics, University Medical Center Regensburg, Regensburg, Germany

2 Department of Gynecology and Obstetrics, Medical Faculty, University Hospital Carl Gustav Carus, TU Dresden, Dresden, Germany

3 Department of Gynecology and Obstetrics, Georg August University Göttingen, University Medicine, Göttingen, Germany
There are theories postulating an increasing risk promoted by polyfollicular ovulation or elevated gonadotropin levels, which are induced by fertility medication. However, the association between ovarian cancer risk and fertility treatments remains controversial [1]. A recently updated Cochrane Review presents new data suggesting that substances used in fertility treatment may cause a slight increase in ovarian cancer risk in subfertile women as compared to the general population or to subfertile women without treatment. However, none of the studies provided high-certainty evidence [2].

Large observational studies show a transient increase in breast cancer risk after the first full-term pregnancy and a long-term protective effect in uni- and biparous women as compared to nulliparous women. Higher transient breast cancer risk was observed especially in women who gave birth at an age of 30-35 years or older [3, 4]. Pregnancies terminated by spontaneous or induced abortion and do not have an impact on breast cancer risk [5]. Higher levels of estrogen and progesterone during pregnancy are discussed 
as underlying mechanisms that cause the transient increase in risk [3].Therefore, it has to be considered that ovarian stimulation leads to high levels of estrogen and progesterone as well.

According to a recent review published in 2017, most studies do not show an elevated risk for breast cancer in women undergoing fertility treatment as compared to parous and nonporous women without fertility treatment. However, uncertainty remains particularly when the fertility treatment is started at a young age or contains a high number of clomiphene citrate cycles [1].

Mutations in BRCA1 and BRCA2 genes represent a significant independent risk factor for ovarian and breast cancer. The cumulative risk to the age of 80 years for ovarian cancer is elevated up to $44 \%$ in BRCAl and up to $17 \%$ in $B R C A 2$ mutation carriers. Breast cancer risk is elevated up to $72 \%$ for $B R C A 1$ and up to $69 \%$ for BRCA2 mutation carriers, respectively [6]. There are theories indicating that deficient $B R C A$ function might lead to reduced fertility due to diminished ovarian reserve [7]. Similarly, more recent studies suggest a negative impact of $B R C A$ mutations on fertility treatment response rates [8]. This hypothesis and the possibly occurring psychological urge to fulfill family planning in a certain time in order to schedule risk-reducing surgery procedures contribute to the fact that fertility treatment and cancer risk is of particular interest for the subgroup of $B R C A 1 / 2$ mutation carriers. This applies especially for $B R C A 1 / 2$ mutation carriers with a previous history of malignant disease. For the general population, the recent data indicate that the long-term outcome of women with pregnancy after breast cancer is not inferior to the outcome of nonpregnant matched controls [9]. This seems to be independent of hormone receptor status. The current data suggest safety for pregnancy after breast cancer also in BRCA1/2 mutation carriers [10]. However, the data are still inhomogeneous and scarce, a higher risk for recurrence cannot be excluded. Therefore, fertility protection and fertility treatment of healthy carriers of mutations in BRCA1 and BRCA2 as well as of carriers after breast cancer therapy are gaining importance. In this review, we analyze the published data on fertility treatments and their association with primary ovarian and breast cancer risk and risk for recurrence in $B R C A 1 / 2$ mutation carriers.

\section{Materials and methods}

A search for original articles was run from 1995 to 2018 on PubMed. The MeSH used for the search were: ("IVF" or "ovarian hyperstimulation") and ("BRCA mutation"), and ("IVF" or "ovarian hyperstimulation") and ("breast cancer" or "ovarian cancer"). The search created 12, 0, 99, 42,75 , and 28 hits, respectively. The hits were searched for relevance, clinical trials, reviews, and meta analyses. We also conducted a search within the Cochrane library. There were no Cochrane reviews available on fertility treatments and risk of cancer in BRCA1/2 mutation carriers.

We found four original publications, two of which regarding ovarian cancer risk, and two regarding breast cancer risk.

\section{Results}

\section{Ovarian cancer}

We found one retrospective cohort study and one case-control study regarding the association of fertility treatments and ovarian cancer risk in $B R C A 1 / 2$ mutation carriers to include in our review (Table 1).

Perri et al. conducted a cohort study with Jewish Israeli women undergoing genetic counseling because of family history of ovarian cancer [11]. They included a total of $718 B R C A 1$ and 331 BRCA2 mutation carriers. 139 of the $B R C A 1$ and 33 of the BRCA2 mutation carriers were diagnosed with ovarian cancer. Furthermore, three healthy $B R C A 1+2$ mutation carriers and three carriers of unknown mutations with ovarian cancer as well as 18 carriers of unknown mutations without ovarian cancer were included. Among the included women, 164 reported undergoing fertility treatments. Among those were $105 B R C A 1,54 B R C A 2$ and one $B R C A l+2$ mutation carriers and four patients with unknown mutations. The treatments included medications containing clomiphene citrate (CC) or gonadotropins (GN) in 82 and 69 of the participants, respectively. Three patients in the $\mathrm{CC}$ and one in the GN group were diagnosed with ovarian cancer. 66 participants reported in vitro fertilization (IVF) and 50 received a combination of those treatments. 4 of the IVF and 10 of the mixed treatment group had ovarian cancer, respectively. The mean age of the women included was 47.1 years in the group with and 50.4 years in the group without fertility treatment. The patients with ovarian cancer had a mean age of 53.6 and the patients without ovarian cancer of 49.1 years. $13.4 \%$ of the group with and $10.1 \%$ of the group without fertility treatment had undergone prophylactic bilateral salpingo-oophorectomy (PBSO) at a mean age of 44.7 and 46.9 years in the group with and without fertility treatment, respectively. In the multivariate age-adjusted analysis, the study found no association between ovarian cancer and fertility treatments for BRCAl $(\mathrm{OR}=0.81 ; 95 \%$ CI 0.43-1.53) and BRCA2 mutation carriers $(\mathrm{OR}=1.01$; 95\% CI 0.31-3.30). No association was found also after adjustment for the different fertility treatments, i.e., CC, GN, and IVF. Dosage, number, and duration of the different treatments were not investigated, and information on the cause of infertility was not given. 
Table 1 Fertility treatments and risk of ovarian cancer in $B R C A$ mutation carriers
Study/study design/Oxford Center of EvidenceBased Medicine (OCEBM) level of evidence (LOE)

Perri et al. [11]

Retrospective cohort study LOE $2 b$

Gronwald et al. [12]

Case-control study LOE $3 b$
Number

Results

Cases: 139 BRCA1, 33 BRCA2, 3
unknown
Controls: 579 BRCA1, 298
BRCA2, 3 BRCA1+2, 18
unknown

No association

BRCA1: Any

treatment:

$\mathrm{OR}=0.81 ; 95 \%$

CI $0.43-1.53$

IVF: $\mathrm{OR}=0.82$;

95\% CI

$0.23-2.93$

GN: $\mathrm{OR}=0.50$;

95\% CI

$0.06-4.26$

Mixed treat-

ments: $\mathrm{OR}=1.8$;

95\% CI

$0.78-4.15$

BRCA2: Any

treatment:

$\mathrm{OR}=1.01 ; 95 \%$

CI $0.31-3.30$

IVF: $\mathrm{OR}=1,81$,

95\% CI

$0.20-16.48$

$\mathrm{CC}: \mathrm{OR}=3.14$,

95\% CI

$0.57-17.41$

Mixed treat-

ments:

$\mathrm{OR}=0.77 ; 95 \%$

CI 0.09-6.95

Cases: 791 BRCA1, 150 BRCA2

No association

Controls: 791 BRCA1, 150 BRCA2

$\mathrm{OR}=0.66 ; 95 \%$

CI 0.18-2.33,

$p=0.52$

IUI: $\mathrm{OR}=0.83$;

95\% CI $0.22-$

$3.13, p=0.79$

SERM:

$\mathrm{OR}=0.55 ; 95 \%$

CI 0.27-1.10,

$p=0.09$

$\mathrm{GN}: \mathrm{OR}=1.35$;

95\% CI 0.11-

16.62, $p=0.82$

Other: $\mathrm{OR}=1.00$

(95\% CI 0.25-

$4.00, p=1.0$

Gronwald et al. included 791 BRCA1 and 150 BRCA2 case-control pairs in their study. In total, 11 participants had undergone IVF and 9 intrauterine insemination (IUI) [12]. 34 had been treated with selective estrogen receptor modulators (SERM), two with gonadotropins (GN) and 8 with other fertility medications. Among the ovarian cancer cases, 4 had undergone IVF and IUI each. 12 of the ovarian cancer patients had used SERM, two GN and 4 other fertility medication. The mean age was 64 and 63 years for cases and controls. The study found no association between ovarian cancer and IVF treatment $(\mathrm{OR}=0.66 ; 95 \% \mathrm{CI} 0.18-2.33$, $p=0.52)$ or IUI $(\mathrm{OR}=0.83 ; 95 \%$ CI $0.22-3.13, p=0.79)$ in $B R C A$ mutation carriers, although the confidence intervals were wide. Likewise, no association was found between ovarian cancer and the use of fertility medication. ORs were 0.55 (95\% CI 0.27-1.10, $p=0.09$ ) for SERM, 1.35 (95\% CI $0.11-16.62, p=0.82$ ) for $\mathrm{GN}$ and 1.00 (95\% CI 0.25-4.00, $p=1.0$ ) for other medication. Separate analyses for BRCAI 
and $B R C A 2$ carriers were not provided. Dosage, number, and duration of the different treatments were not examined and the cause of infertility was not provided.

\section{Breast cancer}

We found one case-control study on the association between fertility treatment and breast cancer risk in $B R C A$ mutation carriers and one prospective cohort study on the long-term safety of medication used for fertility preservation in women already diagnosed with breast cancer (Table 2).

Kotsopoulos et al. conducted a matched case-control study that included 1054 pairs of BRCA1 and 326 pairs of $B R C A 2$ mutation carriers [13]. In total, 9 of the cases and 11 of the controls had undergone IVF. 61 of the cases and 56 of the controls reported ever use of fertility medication. Among those, 44\% (24 cases and 27 controls) had used medication-containing clomiphene citrate (CC), 22\% (16 cases and 10 controls) medication-containing gonadotropins (GN), and $8 \%$ other fertility medication, such as bromocriptine, a combination of various drugs or estrogen. For $26 \%$, further information on the fertility medication was missing. The mean age was 46.0 years for cases and 46.2 years for controls. Women who had undergone bilateral mastectomy were excluded. The study did not find an association between the risk of breast cancer and IVF treatment $(\mathrm{OR}=0.98 ; 95 \%$ $\mathrm{CI}=0.39-2.45)$ in $B R C A 1 / 2$ mutation carriers. No association was found when adjusted for $B R C A l(\mathrm{OR}=0.99 ; 95 \%$ CI 0.35-2.76) and BRCA2 (OR $=0.88$; 95\% CI 0.12-6.52) mutation. Likewise, the study did not find an increase in risk associated with the use of fertility medication $(\mathrm{OR}=1.21$; 95\% $\mathrm{CI}=0.81-1.82)$ in $B R C A 1 / 2$ mutation carriers. The results were similar for $B R C A l(\mathrm{OR}=1.22 ; 95 \% \mathrm{CI}$ 0.76-1.94) and BRCA2 (OR $=1.25$; 95\% CI 0.56-2.78) mutation carriers. These results were not significantly affected by stratification for parity. When adjusted for the type of fertility medication, the study found no association between breast cancer risk and medication-containing clomiphene citrate $(\mathrm{OR}=0.96$; $95 \%$ CI $0.54-1.72, p=0.89)$ in BRCA1/2 mutation carriers. A possible adverse effect was observed for $\mathrm{GN}$ containing fertility medication $(\mathrm{OR}=2.32$; 95\% CI $0.91-5.95, p=0.08$ ). However, only few women were exposed and the effect was not statistically relevant. Dosage, number, and duration of the different treatments as well were not provided and possible effects of different causes of infertility were not examined.

Kim et al. included 337 breast cancer patients in a prospective cohort study to investigate the safety of letrozole

Table 2 Fertility treatments and risk of breast cancer in $B R C A$ mutation carriers

\begin{tabular}{llc}
\hline Study/study design/Oxford Center of Evidence-Based Medicine & Number & Results \\
(OCEBM) level of evidence (LOE) & &
\end{tabular}
(OCEBM) level of evidence (LOE)

Kotsopoulos et al. [20]

Case-control study

LOE $3 b$
Cases: 1054 BRCA1, 326 BRCA2

Controls: 1054 BRCA1, 326 BRCA2

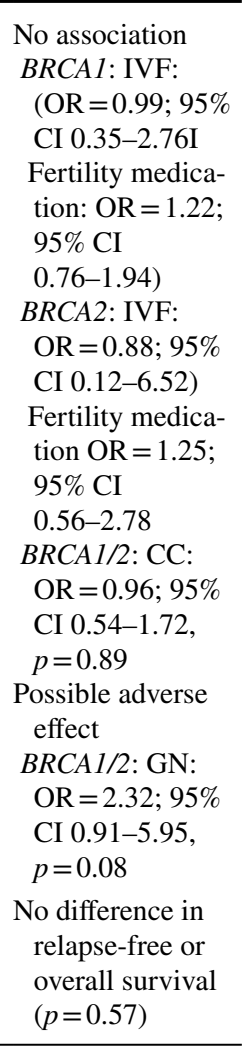

Kim et. al. [14]

Prospective cohort study

LOE $1 b$
Cases (FP): 26 BRCA1/2

Controls: 21 BRCA1/2

In total: 28 BRCA1, 18 BRCA2, 1 BRCA1+2 with breast cancer diagnosis 
and gonadotropin stimulation for fertility preservation (FP) [14]. 120 women underwent FP before chemotherapy, the rest served as control group. In total, the study included $28 B R C A 1,18 B R C A 2$ carriers and one carrier of both a mutation in BRCA1 and BRCA2. 26 of the mutation carriers underwent FP. The mean gonadotropin dose for ovarian stimulation was $2511.0 \pm 1557.0 \mathrm{IU}$. The mean follow-up after diagnosis was 5 years in the FP group and 6.9 years for controls. The study did not find a difference in the relapsefree or overall survival between the FP and the control group using the Kaplan-Meier method ( $p=0.57)$. Also, tumor size, grading, involvement of lymph nodes, hormone receptor status and HER-2/neu overexpression were examined. No significant effect was found for these variables when FP and control group were compared. Molecular testing was not available for all participants and numbers were low. Information on risk-reducing salpingo-oophorectomy during followup was not collected and separate analyses for BRCAl and $B R C A 2$ mutation carriers were not provided.

\section{Discussion}

Studies on the cancer risk of fertility treatment in women with mutations in the genes $B R C A 1$ and $B R C A 2$ are sparse. However, the clinical need for counseling on this subject is highly relevant. In women without evidence for hereditary breast or ovarian cancer, most of the existing studies do not show an elevated risk for breast cancer after clomiphene or gonadotropin treatment [1]. Also, it is unlikely that these medications lead to increases of ovarian cancer risk.

For ovarian or breast cancer, the four available studies show no association between fertility treatments and risk of cancer in BRCA1/2 mutation carriers. For breast cancer, one of the studies shows a possible non-significant adverse effect for gonadotropin-containing fertility medication, while another study does not confirm this effect [13, 14]. Overall, the data on the safety of fertility treatments in BRCA mutation carriers are limited. Most of the studies are retrospective and study populations were often small, especially regarding $B R C A 2$ mutation carriers. Study designs were different and only one of the studies included BRCA1/2 mutation carriers already diagnosed with breast cancer [14]. None of the published studies investigated a possible effect of dosage, number, and duration of the different treatments. Likewise, the causes of infertility and a possible impact on cancer risk were not examined. Only one of the included studies included information on different breast cancer subtypes [14].

Prospective data from clinical registries are needed to further investigate the safety of fertility treatments in BRCA1/2 mutation carriers and to be able to evaluate a possible impact of dosage, number, and duration of the different treatments. Longer follow-up periods are required. Counselling and treatment of families with hereditary breast and ovarian cancer under trial conditions is therefore strongly recommended. The German Consortium Hereditary Breast and Ovarian Cancer (GC-HBOC) offers all families with known or suspected hereditary risk to be registered in the HerediCaRe study, which is supported by a grant of the German Ministry of Education and Research. The prospective follow up within this registry and the international cooperations on research about genetic and non-genetic risk factors will allow a more knowledge-based view on potential risks and clinical options of prevention in the future $[2,6,15,16]$. BRCA1/2associated breast cancer differs from cancer that arises in non-carriers. In breast cancer, an onset about 20 years earlier (44 years in BRCAl vs 64 years for sporadic BC) and a higher number of hormone receptor negative cancers are the main differences $[6,17,18]$. And even for BRCA1/2associated ovarian cancer, which is very close to sporadic ovarian cancer in terms of histopathology, the age of onset is at least 10 years earlier (59 years in BRCAl vs 69 years for sporadic OC) $[6,17,18]$. Exposure of lifestyle factors at different ages might influence cancer risk in carriers in a different way than expected. Until association of lifestyle and cancer risk is not fully understood, it is difficult to exclude an elevated cancer risk. Intensified research in a small, well characterized group of women such as BRCA1/2 mutation carriers will help elucidate tumorigenesis also for the general population. An example of how evidence in the field of hereditary breast and ovarian cancer changes understanding and therapy of other cancers is the targeted therapy with PARP inhibitors [19].

Based on the existing studies, BRCA1/2 mutation carriers should be not be excluded from utilization of fertility treatments, but at the same time take part in a clinical registry to follow up cancer history. More data will be needed since possible increases in cancer risk cannot be excluded at this time. Therefore, women with BRCAl/2 mutations who consider fertility treatment have to be informed about the limited data. They have to be informed about possible increases in cancer risk associated with different treatments. Fertility treatments have to be used with care in this specific subgroup.

Acknowledgments Open Access funding provided by Projekt DEAL.

Author contribution D. Huber: literature search, data analysis, manuscript writing. S. Seitz: data analysis, manuscript writing. K. Kast: manuscript editing, G. Emons: manuscript editing. O. Ortmann: project development, manuscript editing. 


\section{Compliance with ethical standards}

Conflict of interest The authors declare that they have no conflict of interest.

Open Access This article is licensed under a Creative Commons Attribution 4.0 International License, which permits use, sharing, adaptation, distribution and reproduction in any medium or format, as long as you give appropriate credit to the original author(s) and the source, provide a link to the Creative Commons licence, and indicate if changes were made. The images or other third party material in this article are included in the article's Creative Commons licence, unless indicated otherwise in a credit line to the material. If material is not included in the article's Creative Commons licence and your intended use is not permitted by statutory regulation or exceeds the permitted use, you will need to obtain permission directly from the copyright holder. To view a copy of this licence, visit http://creativecommons.org/licenses/by/4.0/.

\section{References}

1. Kroener L, Dumesic D, Al-Safi Z (2017) Use of fertility medications and cancer risk: a review and update. Curr Opin Obstet Gynecol 29(4):195-201

2. Rizzuto I, Behrens RF, Smith LA (2019) Risk of ovarian cancer in women treated with ovarian stimulating drugs for infertility. Cochrane Database Syst Rev 6:CD008215

3. Lambe M, Hsieh C, Trichopoulos D, Ekbom A, Pavia M, Adami HO (1994) Transient increase in the risk of breast cancer after giving birth. N Engl J Med 331(1):5-9

4. Albrektsen G, Heuch I, Hansen S, Kvåle G (2005) Breast cancer risk by age at birth, time since birth and time intervals between births: exploring interaction effects. Br J Cancer 92(1):167-175

5. Beral V, Doll R, Hermon C, Peto R, Reeves G (2008) Ovarian cancer and oral contraceptives: collaborative reanalysis of data from 45 epidemiological studies including 23,257 women with ovarian cancer and 87,303 controls. Lancet 371(9609):303-314

6. Kuchenbaecker KB, Hopper JL, Barnes DR, Phillips K-A, Mooij TM, Roos-Blom M-J et al (2017) Risks of breast, ovarian, and contralateral breast cancer for $B R C A 1$ and $B R C A 2$ mutation carriers. JAMA 317(23):2402-2416

7. Oktay K, Kim JY, Barad D, Babayev SN (2010) Association of BRCA1 mutations with occult primary ovarian insufficiency: a possible explanation for the link between infertility and breast/ ovarian cancer risks. J Clin Oncol 28(2):240-244

8. Lambertini M, Goldrat O, Ferreira AR, Dechene J, Azim HA, Desir J et al (2018) Reproductive potential and performance of fertility preservation strategies in BRCA-mutated breast cancer patients. Ann Oncol 29(1):237-243

9. Lambertini M, Kroman N, Ameye L, Cordoba O, Pinto A, Benedetti $\mathrm{G}$ et al (2018) Long-term safety of pregnancy following breast cancer according to estrogen receptor status. J Natl Cancer Inst 110(4):426-429
10. Lambertini M, Ameye L, Hamy A-S, Zingarello A, Poorvu PD, Carrasco E, Grinshpun A, Han S, Rousset-Jablonski C, Ferrari A, Paluch-Shimon S, Cortesi L, Senechal C, Miolo G, Pogoda K, Perez-Fidalgo JA, De Marchis L, Del Mastro L, Peccatori F, Azim HA (2019) Safety of pregnancy following breast cancer (BC) in patients (pts) carrying a $B R C A$ mutation (mBRCA): results of an international cohort study. J Clin Oncol 37(suppl 15):11506

11. Perri T, Lifshitz D, Sadetzki S, Oberman B, Meirow D, BenBaruch $\mathrm{G}$ et al (2015) Fertility treatments and invasive epithelial ovarian cancer risk in Jewish Israeli $B R C A 1$ or $B R C A 2$ mutation carriers. Fertil Steril 103(5):1305-1312

12. Gronwald J, Glass K, Rosen B, Karlan B, Tung N, Neuhausen SL et al (2016) Treatment of infertility does not increase the risk of ovarian cancer among women with a $B R C A 1$ or $B R C A 2$ mutation. Fertil Steril 105(3):781-785

13. Kotsopoulos J, Librach CL, Lubinski J, Gronwald J, Kim-Sing C, Ghadirian P et al (2008) Infertility, treatment of infertility, and the risk of breast cancer among women with $B R C A l$ and BRCA2 mutations: a case-control study. Cancer Causes Control 19(10):1111-1119

14. Kim J, Turan V, Oktay K (2016) Long-term safety of letrozole and gonadotropin stimulation for fertility preservation in women with breast cancer. J Clin Endocrinol Metab 101(4):1364-1371

15. Antoniou AC, Goldgar DE, Andrieu N, Chang-Claude J, Brohet R, Rookus MA et al (2005) A weighted cohort approach for analysing factors modifying disease risks in carriers of high-risk susceptibility genes. Genet Epidemiol 29(1):1-11

16. Kuchenbaecker KB, McGuffog L, Barrowdale D, Lee A, Soucy P, Dennis J et al (2017) Evaluation of polygenic risk scores for breast and ovarian cancer risk prediction in BRCA1 and BRCA2 mutation carriers. J Natl Cancer Inst 109(7):djw302

17. Mavaddat N, Barrowdale D, Andrulis IL, Domchek SM, Eccles D, Nevanlinna $\mathrm{H}$ et al (2012) Pathology of breast and ovarian cancers among $B R C A 1$ and $B R C A 2$ mutation carriers: results from the consortium of investigators of modifiers of BRCA1/2 (CIMBA). Cancer Epidemiol Biomarkers Prev 21(1):134-147

18. Krebs in Deutschland 2011/2012: Eine gemeinsame Veröffentlichung des Robert Koch-Instituts und derGesellschaft der epidemiologischen Krebsregister in Deutschland e. V. [10. Ausgabe]. Berlin; 2015

19. Mirza MR, Monk BJ, Herrstedt J, Oza AM, Mahner S, Redondo A et al (2016) Niraparib maintenance therapy in platinum-sensitive, recurrent ovarian cancer. N Engl J Med 375(22):2154-2164

20. Kotsopoulos J, Lubinski J, Moller P, Lynch HT, Singer CF, Eng $C$ et al (2014) Timing of oral contraceptive use and the risk of breast cancer in BRCA1 mutation carriers. Breast Cancer Res Treat 143(3):579-586

Publisher's Note Springer Nature remains neutral with regard to jurisdictional claims in published maps and institutional affiliations. 